\title{
Desinserción meniscal como causa de bloqueo articular: correlación clínico-radiográfica
}

\author{
Mariana Patricia Márquez Benítez, ${ }^{1}$ Víctor Hugo Aguirre Rodríguez, ${ }^{2}$ \\ Felipe Sosa Fermín, ${ }^{3}$ Fernando Sergio Valero González, ${ }^{4}$ Felipe de Jesús Martínez Ruiz, \\ Julián Sánchez Cortázar, ${ }^{6}$ María de Guadalupe Gómez Pérez ${ }^{7}$
}

\section{Resumen}

Los ligamentos meniscocapsulares son estructuras de fijación del menisco; su lesión contribuye a la hipermovilidad del menisco y su posible desinserción. La resonancia magnética (RM) es el estudio de elección para valorar estas estructuras. La desinserción meniscal se puede confundir con una ruptura meniscal en asa de balde, por lo que habrá que tomar en cuenta este diagnóstico diferencial.

Palabras clave: Desinserción meniscocapsular, ligamentos meniscocapsulares, menisco medial.

\section{INTRODUCCIÓN}

Los meniscos son estructuras fibrocartilaginosas cuyas funciones principales son proteger al cartílago, absorber fuerzas y proporcionar lubricación articular. ${ }^{1}$

Cada menisco se subdivide en cuerno anterior, cuerpo y cuerno posterior. Las prolongaciones que emergen anterior y posteriormente se anclan al platillo tibial y femoral, manteniendo al menisco en su posición habitual. ${ }^{1}$

En estudios cinemáticos de rodillas sanas se han documentado movimientos combinados de rodamiento y

\begin{abstract}
Summary
Meniscocapsular ligaments are meniscal fixation structures; their lesions contribute to meniscal hypermobility and its possible detachment. Its gold standard method of study is magnetic resonance imaging. Meniscal detachment can be mistaken for a bucket-handle tear, and therefore, it should be considered as a differential diagnosis.
\end{abstract}

Key words: Meniscocapsular separation, meniscocapsular ligaments, medial meniscus.

deslizamiento, con desplazamiento posterior del punto de contacto femorotibial conforme se incrementa la flexión. Los meniscos medial y lateral se desplazan posteriormente sobre la meseta tibial durante la flexión de la rodilla. ${ }^{2} \mathrm{El}$ desplazamiento posterior del menisco lateral $(8.2 \pm 3.2$ $\mathrm{mm})$ es mayor que el del medial $(3.3 \pm 1.5 \mathrm{~mm})$; esta asimetría del movimiento entre ambos meniscos establece características biomecánicas en las rodillas que están relacionadas con los patrones de lesión. ${ }^{3}$ Por lo anterior, el menisco medial es menos móvil, con un cuerno posterior más ancho que se va estrechando hacia la parte anterior, ${ }^{1}$ lo que lo hace más susceptible a eventos traumáticos.

\footnotetext{
${ }^{1}$ Residente del postgrado en Resonancia Magnética de Cuerpo Completo del Hospital Ángeles Pedregal.

${ }^{2}$ Cirujano ortopedista. Asociado en Reconstrucción Articular, SC. Cadera y Rodilla, Hospital Ángeles Pedregal.

${ }^{3}$ Residente del postgrado en Resonancia Magnética de Musculoesquelético del Hospital Ángeles Pedregal.

${ }^{4}$ Cirujano ortopedista. Director de Reconstrucción Articular, SC. Hombro y Codo, Hospital Ángeles Pedregal.

${ }^{5}$ Residente del postgrado de Alta Especialidad en Reconstrucción Articular de Hombro y Codo.

6 Profesor adjunto al Curso de Alta Especialidad en Medicina, Resonancia Magnética.

7 Medica radióloga. Directora médica de Resonancia Magnética.
}

Correspondencia:

Dr. Víctor Hugo Aguirre Rodríguez

Correo electrónico: cadera.rodilla.hap@gmail.com

Aceptado: 04-01-2016.

Este artículo puede ser consultado en versión completa en http://www.medigraphic.com/actamedica 
La mayor parte de las características complejas tanto morfológicas como funcionales de la rodilla no son exclusivas de los seres humanos; sin embargo, estas particularidades son las que le confieren la diferencia con el resto de los mamíferos. Los meniscos miden aproximadamente $35 \mathrm{~mm}$ en su eje anteroposterior, y el reborde periférico convexo de cada menisco tiene una longitud de aproximadamente $110 \mathrm{~mm}$, incluyendo la longitud de los ligamentos insercionales (ligamento intermeniscal anterior y ligamento meniscofemoral); los meniscos se fijan en sus bordes periféricos hacia el interior de la cápsula articular en toda su longitud, a excepción de una parte del menisco lateral en la región del tendón poplíteo. ${ }^{4}$ En su punto medio, el menisco medial también tiene una unión firme a la parte profunda del ligamento colateral medial. El ligamento insercional del cuerno anterior del menisco medial tiene forma de abanico y se une a la tibia en la zona de la fosa intercondílea, a unos seis o siete milímetros anterior a la inserción del ligamento cruzado anterior; por ello, son comunes las lesiones conjuntas. ${ }^{4}$

También se han identificado dos ligamentos denominados "ligamentos meniscofemorales", ${ }^{3}$ que se unen al cuerno posterior del menisco medial, con dirección hacia el lado lateral del cóndilo medial del fémur en la escotadura intercondílea. El ligamento meniscofemoral anterior corre por delante del ligamento cruzado posterior (LCP) y se conoce como el "ligamento de Humphrey". El ligamento meniscofemoral posterior corre posterior al LCP y se conoce como el "ligamento de Wrisberg". En resonancia magnética (RM) se pueden documentar uno o ambos ligamentos meniscofemorales en 71 a $83 \%$ de los pacientes. ${ }^{5}$

\section{CASO CLÍNICO}

Masculino de 20 años de edad, quien sufrió una lesión de la rodilla izquierda al estar jugando balompié, realizando movimientos rotacionales con valgo forzado súbito. Previamente a la consulta, acudió con un médico ortopedista, quien realizó maniobras de flexo-extensión forzadas. Luego de ello persistió con dolor y sensación de bloqueo, por lo que acudió a revaloración a la consulta cinco días después de la lesión inicial.

En su primera valoración, presentó marcha claudicante y sensación de bloqueo de la rodilla izquierda. A la exploración física, mostró dolor de intensidad 7/10 en la escala visual análoga (EVA) en la línea articular medial, flexión de $110^{\circ}$, extensión de $10^{\circ}$, con signos meniscales positivos.

En la valoración radiológica se solicitaron proyecciones anteroposterior, lateral a $30^{\circ}$ y resonancia magnética de la rodilla realizadas fuera de esta institución.

Las radiografías no documentaron lesión ósea y el reporte de la RM fue compatible con una imagen en "asa de balde".
La cirugía artroscópica demostró desinserción periférica completa del cuerno anterior, cuerpo y esquina posterior del menisco medial con luxación hacia la escotadura intercondílea, mínima laxitud del ligamento cruzado anterior (LCA) y condromalacia en el compartimento medial. Se realizó: a) reducción de la luxación meniscal, b) reparación del cuerpo y cuerpo posterior usando seis anclas, c) aplicación de un punto con súper sutura, con técnica de fuera-adentro para fijar el cuerno anterior tratando de emular la acción del ligamento anterior intermeniscal; d) sinovectomía parcial, e) condroplastia térmica y mecánica, y f) ligamentoplastia térmica del LCA (Figura 1). Después de la cirugía, se indicaron ejercicios de flexo-extensión con rodillera mecánica bloqueada de cero a 30 grados durante cuatro semanas; posteriormente, se incrementó la flexión a 60 grados. Al completar la sexta semana, se permitió la flexión a 100 grados, complementando la rehabilitación con 10 sesiones de terapia física, con la finalidad de mejorar sus rangos de movilidad, calidad de movimiento, fortalecimiento muscular y propiocepción.

Con los hallazgos de la artroscopia se realizó un análisis retrospectivo de la resonancia magnética, donde se identificó en las proyecciones sagitales ausencia del cuerpo y cuerno anterior del menisco medial, con la presencia del "signo de doble cruzado posterior", así como incremento en el líquido articular (Figura 2). En las proyecciones coronales se observó migración de un fragmento meniscal hacia la región intercondílea, así como desplazamiento del menisco a cinco milímetros del margen medial del platillo tibial medial. En los cortes axiales se identificó luxación completa del menisco hacia la región intercondílea (Figura 3).

Con las herramientas avanzadas de lectura, se realizó análisis multiplanar intencionado de los ligamentos meniscocapsulares, y se identificó pérdida de la tensión y continuidad de las fibras del ligamento meniscocapsular femoral medial y conservación del ligamento meniscocapsular tibial medial en las proyecciones coronal y axial, así como la luxación del menisco medial hacia la región intercondílea en los tres planos, coronal, sagital y axial, lo cual creaba una falsa imagen de doble cuerno posterior y de un fragmento meniscal hacia la escotadura intercondílea (Figura 4).

\section{DISCUSIÓN}

Se ha demostrado que al nacer, todo el menisco se encuentra vascularizado por el plexo capilar periférico; sin embargo, se empieza a desarrollar un área avascular en la zona interna del menisco (zona blanca), y en la segunda década de la vida, los vasos sanguíneos alcanzan únicamente el tercio externo (zona roja), abarcando al final 10 a 30\% de vascularidad total en la edad adulta. La pérdida progresiva de vascularidad puede deberse al efecto de carga sobre el menisco y 

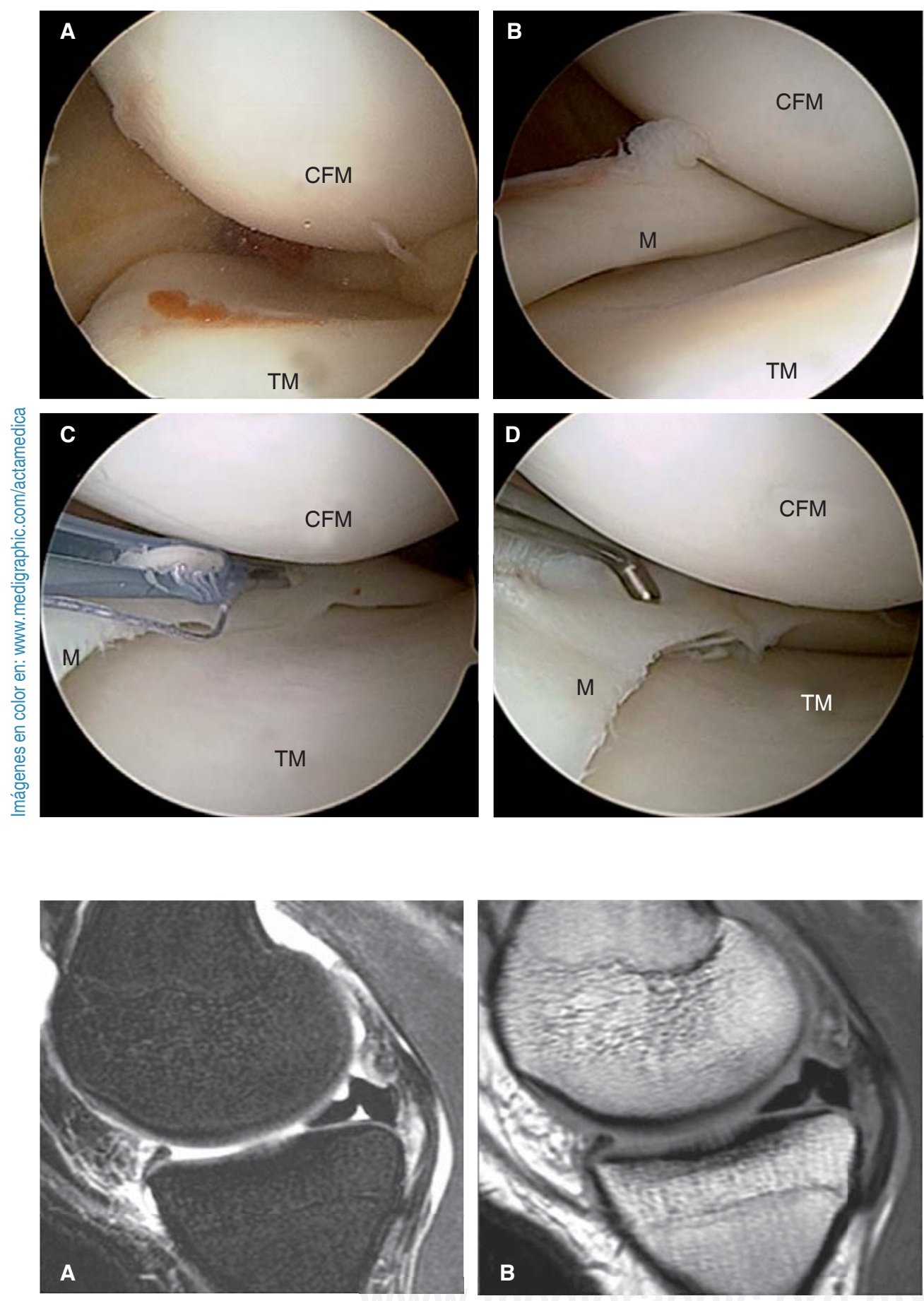

Figura 2.

Proyección sagital. A. Secuencia potenciada en T2 FATSAT y B. secuencia potenciada en T2. Se observa el signo de doble cuerno posterior en el cuerno posterior de menisco medial.

el movimiento de la rodilla; ${ }^{3}$ esta es la fisiopatología por la que algunas fracturas meniscales periféricas curan de forma espontánea, explicando el incremento de intensidad de señal en los meniscos de los niños en la RM. ${ }^{1}$ Estudios anatómicos han demostrado que la irrigación meniscal proviene principalmente de la arteria genicular media. ${ }^{3}$
En las secuencias de RM, los meniscos aparecen como estructuras de baja intensidad de señal, con su conocida forma en corbata de moño en los planos sagitales y en forma triangular en las proyecciones coronales. A pesar de que los meniscos tienen intensidad de señal similar, su morfología macroscópica es diferente. ${ }^{1}$ 

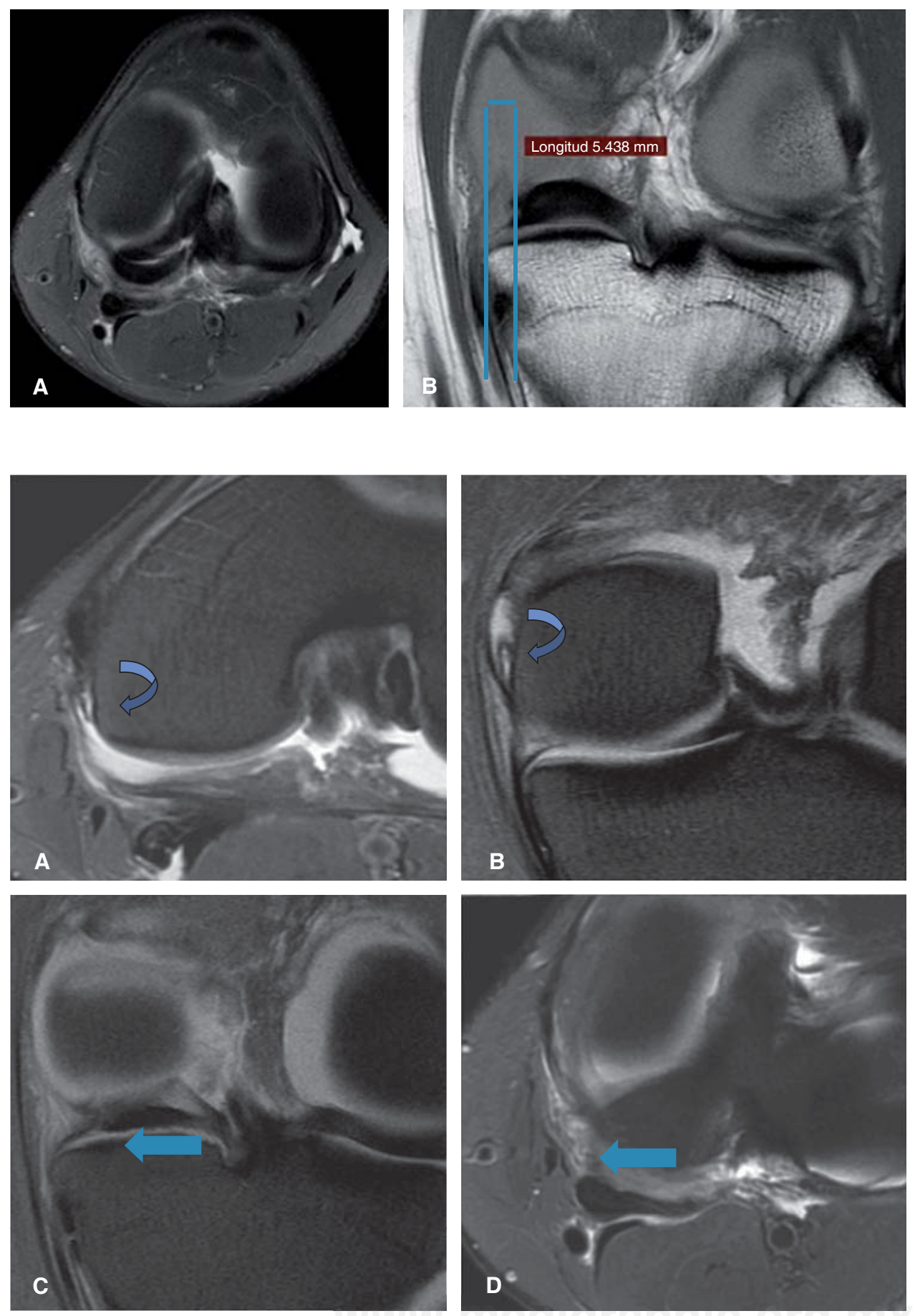

Figura 3.

A. Proyección axial potenciada en T2 FATSAT; se observa luxación del menisco medial hacia la región intercondílea. B. Proyección coronal potenciada en T1, donde se identifica desplazamiento del menisco lateralmente, con una distancia de cinco milímetros del margen medial del platillo tibial medial.

Figura 4.

Imágenes de la región medial de la rodilla. A y B. Corte axial y coronal potenciado en T2 FATSAT, donde se identifica interrupción de las fibras y pérdida de la tensión del ligamento meniscocapsular femoral (flecha curva). C y D. Corte coronal y axial potenciado en T2 FATSAT; se observa integridad de las fibras del ligamento meniscocapsular tibial (flecha recta).
Los ligamentos meniscocapsulares ayudan a la fijación del menisco; se dividen en componentes meniscotibial o coronario y meniscofemoral, que se insertan en la porción posterior de la tibia y del fémur, respectivamente. Estos ligamentos son cortos y contribuyen a la estabilidad del menisco. ${ }^{6}$ Las prolongaciones anteriores del menisco me- dial se insertan, en ocasiones, a lo largo del margen anterior de la tibia y pueden imitar una extrusión.

La separación meniscocapsular puede contribuir a la hipermovilidad del menisco y la inestabilidad de la articulación. ${ }^{7}$ Estos ligamentos están separados de las fibras del ligamento colateral medial por una pequeña 
bursa, que se ve mejor en las imágenes coronales potenciadas en $\mathrm{T} 1 .^{8}$

Un diagnóstico diferencial de la separación meniscocapsular se debe realizar con las fracturas en asa de balde, que son rupturas longitudinales con desplazamiento del fragmento central hacia la escotadura intercondílea; generalmente se presentan en el menisco medial, con una frecuencia siete veces mayor que en el menisco lateral. ${ }^{1}$ Esta fractura ocurre de forma más común en jóvenes deportistas, quienes presentan clínicamente bloqueo articular y/o limitación en la extensión completa.

Las imágenes de RM pueden demostrar pérdida de la morfología habitual del menisco, fragmento hacia la escotadura intercondílea, signo de doble cruzado posterior, doble cuerno anterior, menisco invertido, o solamente un cuerno posterior de menor volumen. ${ }^{9}$

La resonancia magnética es el método de imagen de elección para evaluar la anatomía de los ligamentos meniscocapsulares. ${ }^{6}$

Rubin y sus colaboradores encontraron una pobre correlación entre los hallazgos de la RM para la separación meniscocapsular con los hallazgos de artroscopia, con un valor predictivo positivo (VPP) de $9 \%$ para el menisco medial y de 13\% para el menisco lateral. Esta lesión es más frecuente en el menisco medial que en el lateral. ${ }^{7}$

La separación meniscocapsular permite la hipermovilidad del menisco, que no está necesariamente asociada a un desgarro o fractura meniscal. ${ }^{10}$ La RM tiene sensibilidad y especificidad para detectar una fractura meniscal del 9095\% en la mayoría de los reportes de la literatura.

Los ligamentos meniscocapsulares presentan baja intensidad de señal y son indistinguibles de la porción más profunda del ligamento colateral medial. ${ }^{6}$ Es necesario realizar secuencias con cortes finos (fast spin-echo) ponderadas en $\mathrm{T} 2$, en proyecciones sagitales y coronales, donde se muestre la relación de los meniscos con la meseta tibial y femoral. ${ }^{10}$

En la experiencia de nuestro hospital, las técnicas de imagen usadas para el análisis de los ligamentos y meniscos de las rodillas deberán incluir proyecciones coronales, sagitales y axiales potenciadas en T1, T2 y T2 FATSAT, con cortes finos potenciados en $\mathrm{T} 2$ para la patela y los ligamentos cruzados, con el fin de no obviar el análisis de ninguna de las estructuras de la rodilla.

El radiólogo deberá tener en cuenta a la desinserción meniscal como diagnóstico diferencial de la lesión en asa de balde como causa de bloqueo articular, por lo que habrá que analizar los ligamentos meniscocapsulares en todos los pacientes en quienes se sospechen ambas patologías. El signo del doble cuerno posterior no es específico para la desinserción meniscocapsular, pero deberá hacer pensar en esta entidad.

\section{REFERENCIAS}

1. Nguyen J, De Smet A, Graf B, Rosas H. MR Imaging-based diagnosis and classification of meniscal tears. Radiographics. 2014; 34: 981-999.

2. Yao J, Lancianese SL, Hovinga KR, Lee J, Lerner AL. Magnetic resonance image analysis of meniscal translation and tibio-meniscofemoral contact in deep knee flexion. J Orthop Res. 2008; 26: 673684.

3. Beaufils P, Verdonk R. The meniscus. Heidelberg: Springer-Verlag; 2010.

4. Kohn D, Moreno B. Meniscus insertion anatomy as a basis for meniscus replacement: A morphological cadaveric study. Arthroscopy. 1995; 11: 96-103.

5. Lee BY, Jee WH, Kim JM, Kim BS, Choi KH. Incidence and significance of demonstrating the meniscofemoral ligament on MRI. Br J Radiol. 2000; 73 (867): 271-274.

6. Simão MN, Nogueira-Barbosa MH. Magnetic resonance imaging in the assessment of meniscal anatomic variants and of the perimeniscal ligamentous anatomy: potential interpretation pitfalls. Radiol Bras. 2011; 44: 117-122.

7. Rubin DA, Britton CA, Towers JD, Harner CD. Are MR imaging signs of meniscocapsular separation valid? Radiology. 1996; 201: 829-836.

8. De Maeseneer M, Van Roy F, Lenchik L, Barbaix E, De Ridder F, Osteaux M. Three layers of the medial capsular and supporting structures of the knee: MR imaging anatomic correlation. RadioGraphics. 2000; 20: S83-S89.

9. Ahn JH, Yim SJ, Seo YS, Ko TS, Lee JH. The double flipped meniscus sign: Unusual MRI findings in bucket-handle tear of the lateral meniscus. Knee. 2014; 21 (1): 129-132.

10. Lyle NJ, Sampson MA, Barrett DS. MRI of intermittent meniscal dislocation in the knee. Br J Radiol. 2009; 82 (977): 374-379. 